\title{
MicroRNA-153 functions as a tumor suppressor in gastric cancer via targeting Kruppel-like factor 5
}

\author{
YANLAN OUYANG $^{1}$, WEIJIE YUAN ${ }^{1}$ and SAINAN QIU $^{2}$ \\ Departments of ${ }^{1}$ Gastrointestinal Surgery and ${ }^{2}$ Colorectal Anal Surgery, Xiangya Hospital, \\ Central South University, Changsha, Hunan 410008, P.R. China
}

Received January 29, 2017; Accepted July 20, 2017

DOI: $10.3892 /$ etm.2018.6226

\begin{abstract}
Various microRNAs (miRs) have been demonstrated to serve important roles in gastric cancer (GC). miR-153 in particular has been reported to serve a suppressive role in GC; however, the underlying mechanism remains unclear. In the present study Reverse transcription-quantitative polymerase chain reaction and western blot analysis were used to examine the mRNA and protein expression of Kruppel-like factor 5 . An MTT, wound healing and transwell assay were used to study cell proliferation, migration and invasion, respectively. In the present study, quantitative polymerase chain reaction data indicated that miR-153 was significantly downregulated in GC tissues compared with the adjacent non-tumor tissues. In addition, the reduced expression of miR-153 was significantly associated with GC aggressiveness and poor prognosis of patients. The expression of miR-153 was also reduced in GC cell lines, including KATO III, NCI-N87, SNU-16 and SNU-5, when compared with normal gastric epithelial GES-1 cells. Overexpression of miR-153 in the GC SNU-5 cells by miR-153 mimic transfection significantly inhibited the cell proliferation, migration and invasion. Furthermore, KLF5 was identified as a target gene of miR-153 in SNU-5 cells by bioinformatics prediction. It was observed that KLF5 was significantly upregulated in GC tissues and cell lines, and its expression was negatively regulated by miR-153 in SNU-5 cells. Overexpression of KLF5 impaired the suppressive effects of miR-153 on the proliferation, migration and invasion of SNU-5 cells. In conclusion, the present study demonstrated that miR-153 serves a tumor suppressive role in GC, at least partly, through directly targeting KLF5, thus highlighting the clinical significance of miR-153 in GC.
\end{abstract}

Correspondence to: Dr Sainan Qiu, Department of Colorectal Anal Surgery, Xiangya Hospital, Central South University, 87 Xiangya Road, Changsha, Hunan 410008, P.R. China

E-mail: sainanqiu2015@qq.com

Key words: gastric cancer, microRNA-153, tumor suppressor, Kruppel-like factor 5

\section{Introduction}

Gastric cancer (GC), one of the most common types of human cancer, results in high morbidity and mortality worldwide $(1,2)$. Although strategies involving surgery combined with radiotherapy and chemotherapy have been extensively investigated, patients with advanced GC present poor prognosis (1-4). As the malignant progression of GC is a multistep and multifactorial process, investigation on the molecular mechanism underlying GC growth and metastasis may be helpful for developing effective therapeutic strategies for this disease $(3,4)$.

MicroRNAs (miRs) are small non-coding RNAs containing 22-25 ribonucleic acids, which directly bind to the 3'-untranslated region (3'UTR) of their target genes, causing translation inhibition or mRNA degradation (5). Through negatively mediating the gene expression, miRs participate in the regulation of a variety of cellular biological processes. Certain miRs targeting oncogenes or tumor suppressors have been demonstrated to serve key roles in different types of human cancer (6-9). In addition, previous studies have revealed that numerous miRs, including miR-326 (9), miR-126 (10), miR-145 (11) and miR-506 (12), serve suppressive or oncogenic roles in GC.

Deregulation of miR-153 has recently been observed in several common human tumors, while miR-153 serves a promoting or tumor suppressive role in different cancer types, including GC (13-15). For instance, Zhang et al (13) has reported that miR-153 was downregulated in GC, which promoted the migration and invasion of the GC cells, SGC-7901. Wang and Liu (16) also suggested that miR-153 suppression in GC promoted Snail-mediated cancer metastasis. However, the detailed regulatory mechanism of miR-153 in $\mathrm{GC}$ remains unclear.

Kruppel-like factor 5 (KLF5), a member of the KLF subfamily of zinc finger proteins, is a transcriptional activator that binds directly to a specific recognition motif in the promoters of target genes (17). Recent studies have identified that KLF5 is frequently upregulated in certain human cancer types and functions as an oncogene (14,17-19). Furthermore, Chia et al (20) reported a regulatory crosstalk between KLF5, GATA4 and GATA6 that cooperatively promotes GC development. However, the molecular mechanism underlying KLF5 expression in GC remains largely unknown.

Therefore, the present study aimed to investigate the molecular mechanism of miR-153 underlying the malignant 
progression of GC, as well as examine the involvement of KLF5 in GC.

\section{Materials and methods}

Clinical samples. The current study was approved by the Ethical Committee of Xiangya Hospital, Central South University (Changsha, China). A total of 83 GC tissues and their matched adjacent non-tumor tissues were collected at the Department of Gastrointestinal Surgery of Xiangya Hospital between March 2011 and April 2012, and informed consent were obtained from the patients. The $83 \mathrm{GC}$ patients included 50 male and 33 female, with an age range of 47-82 years and a mean age of 64.7 years. In addition, 42 of the patients with GC were at TNM stage I-II and 41 patients were at TNM stage III-IV (21). These GC patients were divided into low and high miR-153 expression groups based on the mean expression value (1.03) as the cutoff value. The tissues were immediately snap-frozen in liquid nitrogen following surgery and stored at $-80^{\circ} \mathrm{C}$ prior to use. The clinical information of these patients is summarized in Table I.

Cell culture. Human GC cell lines, including KATO III, NCI-N87, SNU-16 and SNU-5, and normal gastric mucosa epithelial GES-1 cells were purchased from the Cell Bank of Central South University. Cells were cultured in Dulbecco's modified Eagle's medium (DMEM; Thermo Fisher Scientific, Inc., Waltham, MA, USA) with $10 \%$ fetal bovine serum (FBS; Thermo Fisher Scientific, Inc.) at $37^{\circ} \mathrm{C}$ in a humidified incubator containing $5 \% \mathrm{CO}_{2}$.

Reverse transcription-quantitative polymerase chain reaction (RT-qPCR). Total RNA was extracted using TRIzol Reagent (Thermo Fisher Scientific, Inc.), according to the manufacturer's protocol. The total RNA was then converted into cDNA using an miRNA Reverse Transcription kit (Thermo Fisher Scientific, Inc.), according to the manufacturer's instructions. The expression of mRNA was then examined using a SYBR Green qPCR Assay kit (Thermo Fisher Scientific, Inc.) on a thermocycler (ABI 7300 plus; Thermo Fisher Scientific, Inc.), while the expression of miR was determined using an miRNA qPCR Detection kit (GeneCopoeia, Inc., Rockville, MD, USA), according to the manufacturer's protocol. GAPDH or U6 was used as the internal reference for the determination of mRNA or miR expression, respectively. The primers sequences used in qPCR were as follows: KLF5 forward, 5'-CCTGGTCCAGACAAGATGTGA-3', and reverse, 5'-GAA CTGGTCTACGACTGAGGC-3'; GAPDH forward, 5'-GGA GCGAGATCCCTCCAAAAT-3', and reverse, 5'-GGCTGT TGTCATACTTCTCATGG-3'. The primers for miR-153 (cat. no. SG-has-miR-153) and U6 (cat. no. SG-U6) were obtained from Shenzhen Hua Anping Hong Biological Technology Co., Ltd. (Shenzhen, China). The qPCR reaction was performed at $95^{\circ} \mathrm{C}$ for $5 \mathrm{~min}$, followed by 45 cycles of denaturation at $95^{\circ} \mathrm{C}$ for $30 \mathrm{sec}$ and annealing/elongation step at $60^{\circ} \mathrm{C}$ for $30 \mathrm{sec}$. The relative expression was analyzed by the $2^{-\Delta \Delta \mathrm{Cq}}$ method (22).

Cell transfection. SNU-5 cells were transfected with scrambled miR mimic (miR-NC), miR-153 mimic, negative control (NC) inhibitor or miR-153 inhibitor, or co-transfected with
miR-153 inhibitor and pcDNA3.1 vector, or with miR-153 inhibitor and pcDNA3.1-KLF5 plasmid (all generated from Yearthbio, Changsha, China). Transfection was performed using Lipofectamine 2000 (Thermo Fisher Scientific) at $37^{\circ} \mathrm{C}$, according to the manufacturer's protocol. Following transfection for $48 \mathrm{~h}$, the mRNA or protein expression levels of KLF5, miR-153 and the corresponding internal controls were determined using RT-qPCR or western blot analysis, respectively.

Cell proliferation analysis. An MTT assay was conducted for the analysis of cell proliferation. Briefly, SNU-5 cells $\left(5 \times 10^{4}\right.$ cells per well) were plated into a 96 -well plate and cultured at $37^{\circ} \mathrm{C}$ with $5 \% \mathrm{CO}_{2}$ for $0,12,24,48$ or $72 \mathrm{~h}$. Subsequently, $20 \mu \mathrm{l}$ of MTT $(5 \mathrm{mg} / \mathrm{ml}$; Sigma-Aldrich; Merck, Darmstadt, Germany) was added and incubated at $37^{\circ} \mathrm{C}$ for $4 \mathrm{~h}$, followed by addition of $150 \mu \mathrm{l}$ of dimethyl sulfoxide (Sigma-Aldrich; Merck). Following incubation at room temperature for $10 \mathrm{~min}$, formazan production was detected by determining the optical density at $570 \mathrm{~nm}$ using an enzyme immunoassay analyzer (Typhoon 8600; GE Healthcare, Chicago, IL, USA).

Cell migration analysis. For the analysis of cell migration, a wound healing assay was conducted. Briefly, SNU-5 cells $\left(2 \times 10^{5}\right.$ cells per well) were seeded into a 24 -well plate and cultured to full confluence. Next, a wound was formed using a plastic cell scraper, and then cells were washed using phosphate-buffered saline (PBS). Subsequent to incubation in DMEM without FBS at $37^{\circ} \mathrm{C}$ for $24 \mathrm{~h}$, the medium was replaced with DMEM containing $10 \% \mathrm{FBS}$, and then cultured at $37^{\circ} \mathrm{C}$ for a further $48 \mathrm{~h}$. Finally, the wounds were observed under a microscope (Nikon Corp., Tokyo, Japan).

Cell invasion analysis. For investigation of cell invasion, a Transwell assay was performed. Briefly, the SNU-5 cell suspension $\left(1 \times 10^{5}\right.$ cells per well) was added into the upper chamber that was pre-coated with Matrigel (Chemicon; EMD Millipore, Billerica, MA, USA), while $300 \mu$ l DMEM containing $10 \%$ FBS was added into the lower chamber. Following incubation at $37^{\circ} \mathrm{C}$ for $24 \mathrm{~h}$, the cells on the interior of the inserts were removed using a cotton-tipped swab. Cells on the lower surface of the membrane were stained with gentian violet (Sigma-Aldrich; Merck), and then rinsed by water, and dried in air. Invading cells were counted under a microscope (Olympus Corp., Tokyo, Japan).

Western blot analysis. SNU-5 cells were lysed in cold radioimmunoprecipitation assay buffer (Thermo Fisher Scientific, Inc.), and the protein concentration was determined using a Bicinchoninic Acid Protein Assay kit (Pierce; Thermo Fisher Scientific, Inc.). Protein was separated by $10 \%$ SDS-PAGE and then transferred to a polyvinylidene difluoride (PVDF) membrane (Thermo Fisher Scientific, Inc.), which was then blocked in 5\% non-fat milk in PBS (Thermo Fisher Scientific, Inc.) containing 0.1\% Tween-20 (Sigma-Aldrich; Merck) at room temperature for $3 \mathrm{~h}$. Subsequently, the PVDF membrane was incubated with rabbit anti-human polyclonal KLF5 (1:50; cat. no. ab137676; Abcam, Cambridge, MA, USA) or rabbit anti-human GAPDH (1:100; cat. no. ab9485; Abcam) primary 

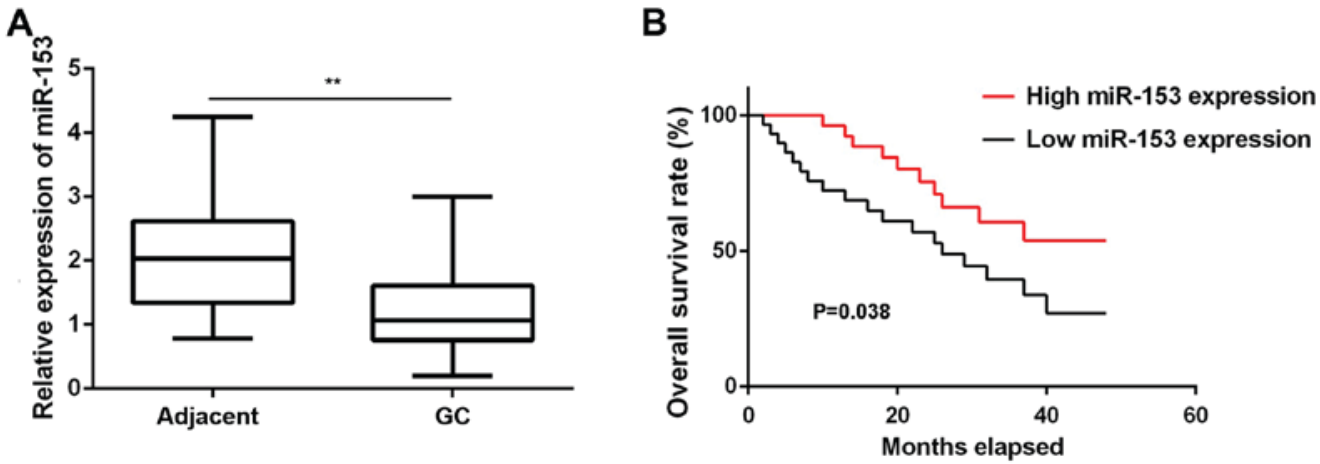

Figure 1. miR-153 is downregulated in GC. (A) Reverse transcription-quantitative polymerase chain reaction was conducted to examine the miR-153 expression in GC tissues and matched adjacent non-tumor tissues. ${ }^{* *} \mathrm{P}<0.01$. (B) Gastric cancer patients with low miR-153 expression presented a shorter survival time when compared with patients with a high expression of miR-153. GC, gastric cancer; miR, microRNA.

antibodies at room temperature for $3 \mathrm{~h}$. Following washing with PBS for $10 \mathrm{~min}$, the PVDF membrane was incubated with a goat anti-rabbit secondary antibody (1:5,000; cat. no. ab7090; Abcam) at room temperature for $1 \mathrm{~h}$. After further washing with PBS for $10 \mathrm{~min}$, the protein bands were detected using an Enhanced Chemiluminescence Western Blotting kit (Pierce; Thermo Fisher Scientific, Inc.), according to the manufacturer's protocols, and then quantified using Image Lab analysis software version 3.1 (Bio-Rad Laboratories, Inc., Hercules, CA, USA).

Bioinformatics prediction. In order to predict the potential target genes of miR-153, the TargetScan (targetscan.org) and PicTar (pictar.mdc-berlin.de) online software were used, according to the manufacturer's instructions. 'MiR-153' was inserted and 'human' was selected. The putative target genes of miR-153 were scanned.

Recombinant vector construction for dual-luciferase reporter assay. The predicted miR-153 binding sites on the 3'UTR of KLF5 were cloned into the pGL 3 vector (Promega Corporation, Madison, WI, USA) and termed wild type (WT)-KLF5-3'UTR. The mutant-type (MT) miR-153 binding sites on the 3'UTR of KLF5 were constructed using a QuikChange Site-Directed Mutagenesis kit (Stratagene; Agilent Technologies, Inc., Santa Clara, CA, USA), in accordance with the manufacturer's protocol. This was also inserted into the pGL3 vector and termed MT-KLF5-3'UTR.

Dual-luciferase reporter gene assay. SNU-5 cells were transfected with $100 \mathrm{nM}$ WT-KLF5-3'UTR or MT-KLF5-3'UTR plasmid, as well as with or without $100 \mathrm{nM} \mathrm{miR-153} \mathrm{mimic.} \mathrm{The}$ luciferase activity was then measured at $48 \mathrm{~h}$ after transfection using the Dual-Luciferase Reporter Assay System (Promega Corporation) on an Lmax II Luminometer (Molecular Devices, LLC, Sunnyvale, CA, USA). The firefly luciferase activity was then normalized to the Renilla luciferase activity.

Statistical analysis. The data in the present study are expressed as the mean \pm standard deviation. Statistical analysis was conducted by Student t-test for comparison between 2 groups or by one-way analysis of variance for comparison of $>2$ groups, using SPSS version 19.0 software (IBM Corp., Armonk, NY,
USA). $\mathrm{P}<0.05$ was considered to indicate a statistically significant difference.

\section{Results}

miR-153 is downregulated in GC, and associated with malignant progression and poor prognosis. In the present study, RT-qPCR data demonstrated that miR-153 was significantly downregulated in GC tissues compared with the adjacent non-tumor tissues (Fig. 1A). These GC patients were divided into low and high miR-153 expression groups based on the mean expression value (1.03) as the cutoff value. In addition, low expression of miR-153 was significantly associated with lymph node metastasis, distant metastasis and advanced TNM stage, although it was not correlated with the patient age, sex, tumor differentiation or tumor size (Table I). Further investigation revealed that the GC patients with low miR-153 expression had a shorter survival time, when compared with those presenting a high miR-153 expression (Fig. 1B). Accordingly, miR-153 downregulation may contribute to the $\mathrm{GC}$ aggressiveness, as well as the poor prognosis of patients.

Overexpression of $m i R-153$ inhibits $G C$ cell proliferation, migration and invasion. The miR-153 expression levels were then examined in several common GC cell lines (KATO III, NCI-N87, SNU-16 and SNU-5), using normal gastric epithelial GES-1 cells as the control. In accordance with the findings in the GC tissues, RT-qPCR data revealed that miR-153 was also significantly downregulated in the four GC cell lines compared with the normal GES-1 cells (Fig. 2A). Since miR-153 expression was downregulated to a greater extent in SNU-5 cells, this cell line was used in subsequent experiments.

SNU-5 cells were then transfected with miR-153 mimic or miR-NC. As shown in Fig. 2B, the miR-153 levels were significantly higher in the miR-153 group compared with the miR-NC group. The study further demonstrated that the overexpression of miR-153 significantly decreased the SNU-5 cell proliferation when compared with the miR-NC group, indicating the suppressive effect of miR-153 on GC cell proliferation (Fig. 2C). Overexpression of miR-153 also reduced the migration and invasion of SNU-5 cells (Fig. 2D and E), suggesting that miR-153 may also inhibit GC metastasis. 
Table I. Association between miR-153 expression and clinicopathological characteristics of gastric cancer patients.

\begin{tabular}{|c|c|c|c|}
\hline Variables & Low miR-153 (n=43) & High miR-153 $(n=40)$ & P-value \\
\hline Age (years) & & & 0.511 \\
\hline$\leq 65$ & 21 & 23 & \\
\hline$>65$ & 22 & 17 & \\
\hline Sex & & & 1.000 \\
\hline Male & 26 & 24 & \\
\hline Female & 17 & 16 & \\
\hline Tumor differentiation & & & 0.080 \\
\hline Well and moderate & 16 & 23 & \\
\hline Poor & 27 & 17 & \\
\hline Tumor size $(\mathrm{cm})$ & & & 0.275 \\
\hline$\leq 5$ & 18 & & \\
\hline$>5$ & 25 & & \\
\hline Lymph node metastasis & & & 0.045 \\
\hline Present & 31 & & \\
\hline Absent & 12 & & \\
\hline Distant metastasis & & & 0.022 \\
\hline Present & 12 & 3 & \\
\hline Absent & 31 & 37 & \\
\hline TNM stage & & & 0.004 \\
\hline I-II & 15 & 27 & \\
\hline III-IV & 28 & 13 & \\
\hline
\end{tabular}

KLF5 is a target gene of miR-153 in GC cells. The potential target genes of miR-153 were further predicted using bioinformatics analysis. As indicated in Fig. 3A, KLF5 was predicted to be a putative target gene of miR-153. To further verify this association, luciferase reporter gene plasmids containing the WT or MT of KLF5 3L-UTR were constructed (Fig. 3B). The dual-luciferase reporter gene assay data indicated that the luciferase activity was significantly decreased following co-transfection with miR-153 mimic and WT-KLF5-3'UTR plasmid, which was eliminated by co-transfection with miR-153 mimic and MT-KLF5-3'UTR plasmid (Fig. 3C). This indicates that miR-153 was able to directly bind to the 3'-UTR of KLF5 mRNA, and therefore, KLF5 is a target gene of miR-153 in GC cells.

KLF5 is upregulated in GC tissues and cells, but negatively regulated by $\mathrm{miR}-153$ in SNU-5 cells. The expression of KLF5 was further examined in GC tissues and cells. As shown in Fig. 4A and B, the mRNA and protein levels of KLF5 were significantly higher in GC tissues as compared with the levels in matched normal adjacent tissues. In addition, these levels were higher in the GC cell lines when compared with the normal gastric epithelial GES-1 cells (Fig. 4C and D).

The regulatory effect of miR-153 on the expression of KLF5 was then examined in SNU-5 cells. As shown in Fig. 5A and B, overexpression of miR-153 significantly reduced the mRNA and protein expression levels of KLF5 in SNU-5 cells, suggesting that miR-153 negatively regulates the expression of KLF5 in GC cells. To further confirm these findings, SNU-5 cells were transfected with an miR-153 inhibitor or NC inhibitor, respectively. Following transfection, the miR-153 levels were significantly reduced in the miR-153 inhibitor group compared with $\mathrm{NC}$ inhibitor group (Fig. 5C). Furthermore, it was observed that knockdown of miR-153 promoted the mRNA and protein expression levels of KLF5 in SNU-5 cells (Fig. 5D and E). Accordingly, KLF5 was suggested to be negatively regulated by miR-153 in GC cells.

KLF5 is a downstream effector in the miR-153-mediated malignant phenotypes of GC cells. According to the aforementioned findings, KLF5 may be involved in the miR-153-mediated malignant phenotypes of SNU-5 cells. To clarify this hypothesis, SNU-5 cells were co-transfected with miR-153 mimic and pcDNA3.1-KLF5 plasmid. Cells that were co-transfected with miR-153 inhibitor and blank pcDNA3.1 vector served as the control group. As indicated in Fig. 6A and B, the mRNA and protein expression levels of KLF5 were significantly increased in the miR-153 + KLF5 group compared with those in the miR-153 + blank group. In addition, the cell proliferation, migration and invasion were significantly upregulated in the miR-153 + KLF5 group compared with the miR-153 + blank group (Fig. 6C-E). These data indicate that the suppressive effects of miR-153 on the proliferation, migration and invasion of SNU-5 cells are possibly through directly targeting KLF5. 
A

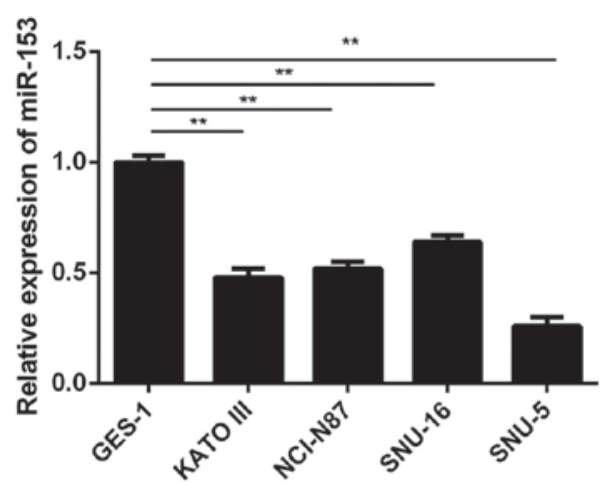

B

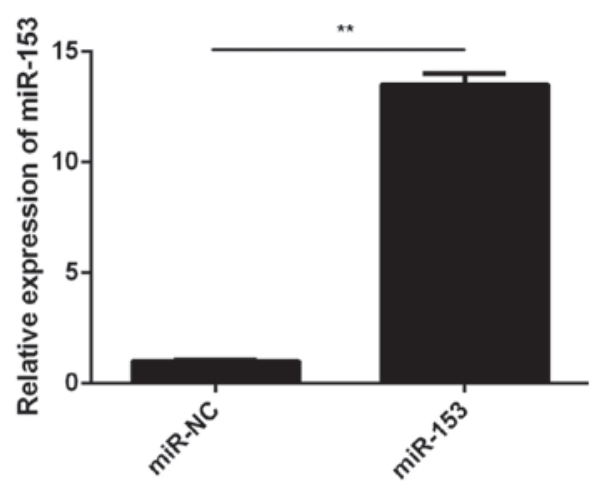

D

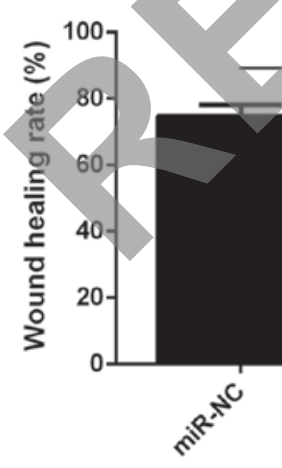

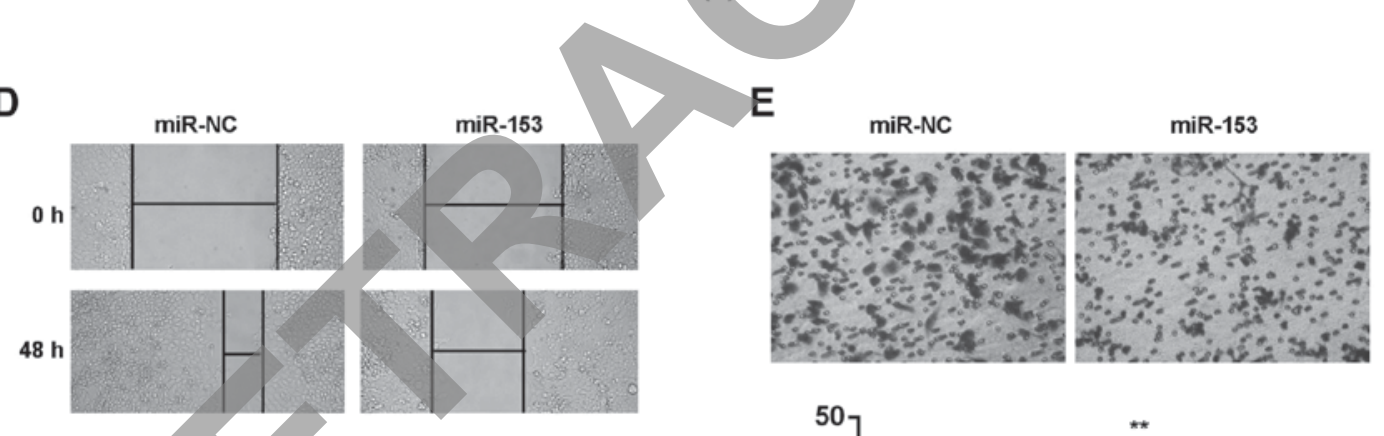

C
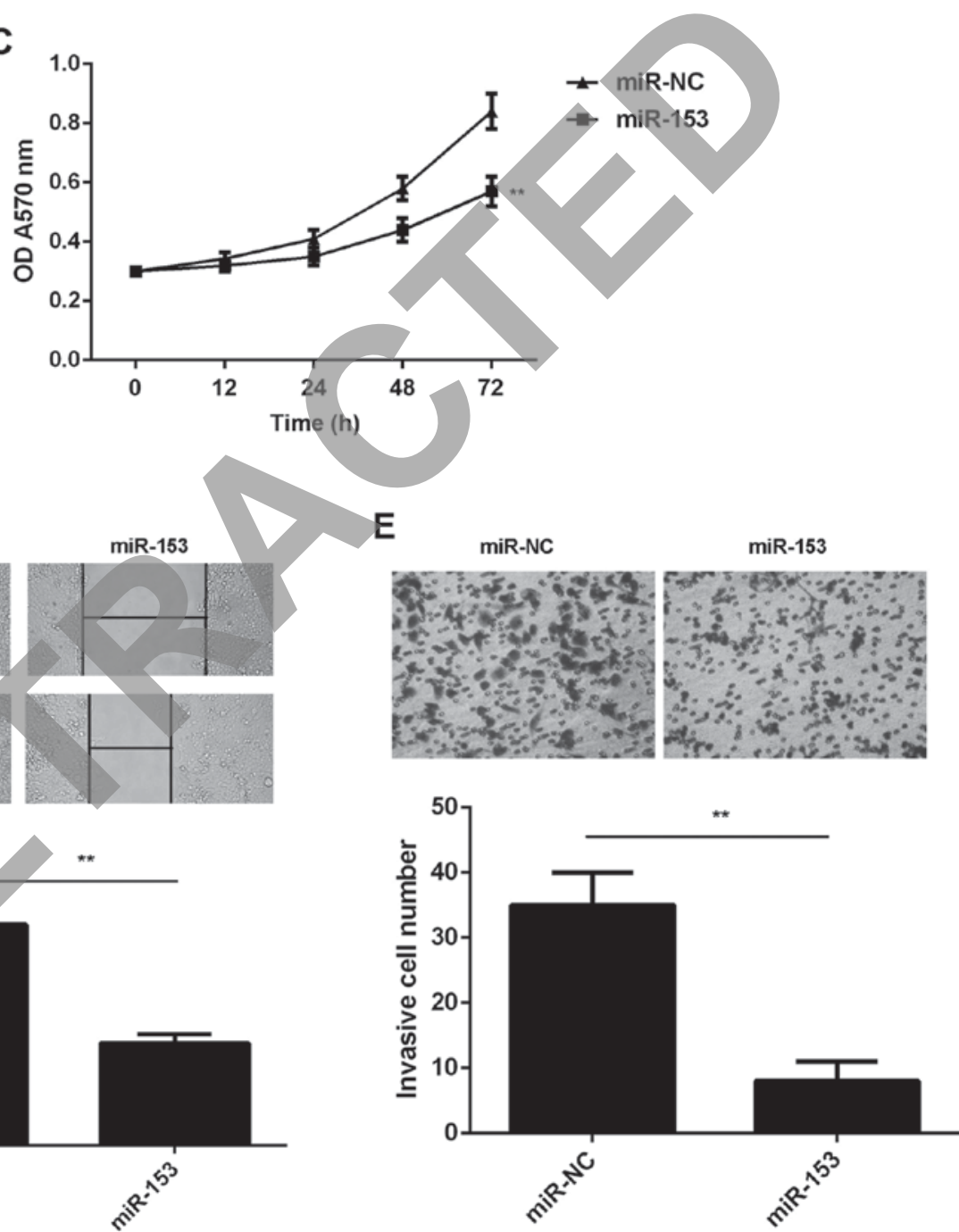

Figure 2. miR-153 inhibits the malignant phenotypes of GC cells. Reverse transcription-quantitative polymerase chain reaction was conducted to examine the miR-153 expression in (A) four GC cell lines (KATO III, NCI-N87, SNU-16 and SNU-5) and normal gastric mucosa epithelial GES-1 cells, and in (B) SNU-5 cells transfected with miR-153 mimic or miR-NC. (C) MTT, (D) wound healing and (E) transwell assays were conducted to examine the proliferation, migration and invasion of SNU-5 cells, respectively. Magnification: Wound healing assay, x40; transwell assay, $\mathrm{x} 400$. ${ }^{* *} \mathrm{P}<0.01$. GC, gastric cancer; miR, microRNA; NC, negative control; OD, optical density.

\section{Discussion}

miR-153 has been suggested to be involved in GC progression (13); however, the molecular mechanism underlying its function remains unclear. In the present study, it was observed that miR-153 was significantly downregulated in GC tissues and cell lines, and this low expression was associated with the GC aggressiveness, as well as poor prognosis of patients. Overexpression of miR-153 induced by mimic transfection was observed to inhibit the SNU-5 cell proliferation, migration and invasion. In addition, KLF5, which was significantly upregulated in GC tissues and cells, was identified as a target 


\begin{tabular}{|c|c|c|c|c|c|c|c|c|}
\hline & & $\begin{array}{l}\text { Predicted consequential pairing of target region (top) } \\
\text { and miRNA (bottom) }\end{array}$ & $\begin{array}{l}\text { Site } \\
\text { type }\end{array}$ & $\begin{array}{c}\text { Context++ } \\
\text { score }\end{array}$ & $\begin{array}{l}\text { Context++ score } \\
\text { percentile }\end{array}$ & $\begin{array}{c}\text { Weighted } \\
\text { context++ score }\end{array}$ & $\begin{array}{l}\text { Conserved brance } \\
\text { length }\end{array}$ & $\mathrm{P}_{\mathrm{cr}}$ \\
\hline $\begin{array}{l}\text { Position 1541-1548 of KLF5 3' UTR } \\
\text { hsa-miR-153-3p }\end{array}$ & $5^{\prime}$ & $\begin{array}{c}\text {... UAUUGUGGUCAUUUCCUAUGCAA... } \\
\text { IIIIII } \\
\text { CUAGUGAAAACACUGAUACGUU }\end{array}$ & 8 mer & -0.39 & 98 & -0.39 & 4.122 & \\
\hline
\end{tabular}

B

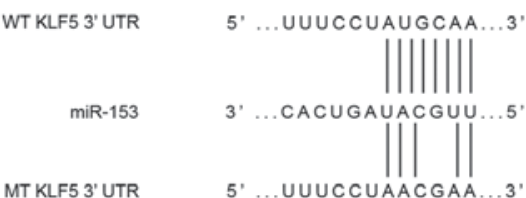

C

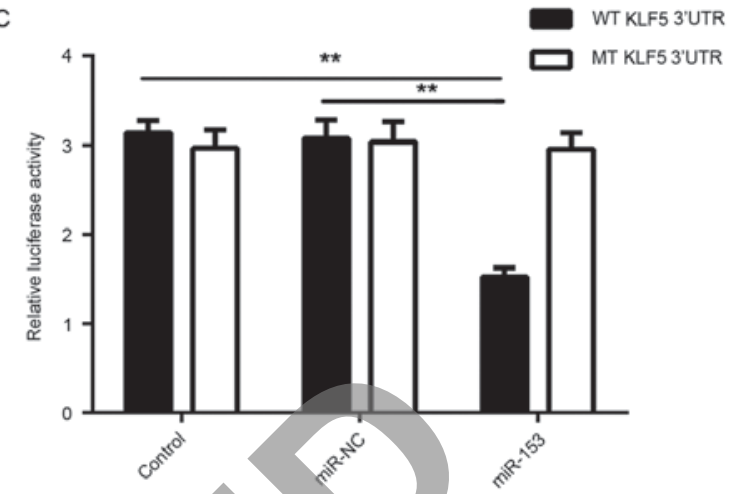

Figure 3. KLF5 is predicted to be a target gene of miR-153. (A) Bioinformatics prediction indicated that KLF5 is a potential target of miR-153. (B) Luciferase reporter gene plasmids containing the WT or MT of KLF5 3'-UTR were generated. (C) Dual-luciferase reporter gene assay data indicated that the luciferase activity was reduced in SNU-5 cells co-transfected with miR-153 mimic and WT-KLF5-3'UTR reporter plasmid, but was unaltered in cells co-transfected with miR-153 mimic and MT-KLF5-3'UTR plasmid. ${ }^{* *}$ P $<0.01$. KLP5, Kruppel-like factor 5; miR, microRNA; WT, wild-type; MT, mutant-type; 3'UTR, 3'-untranslated region.

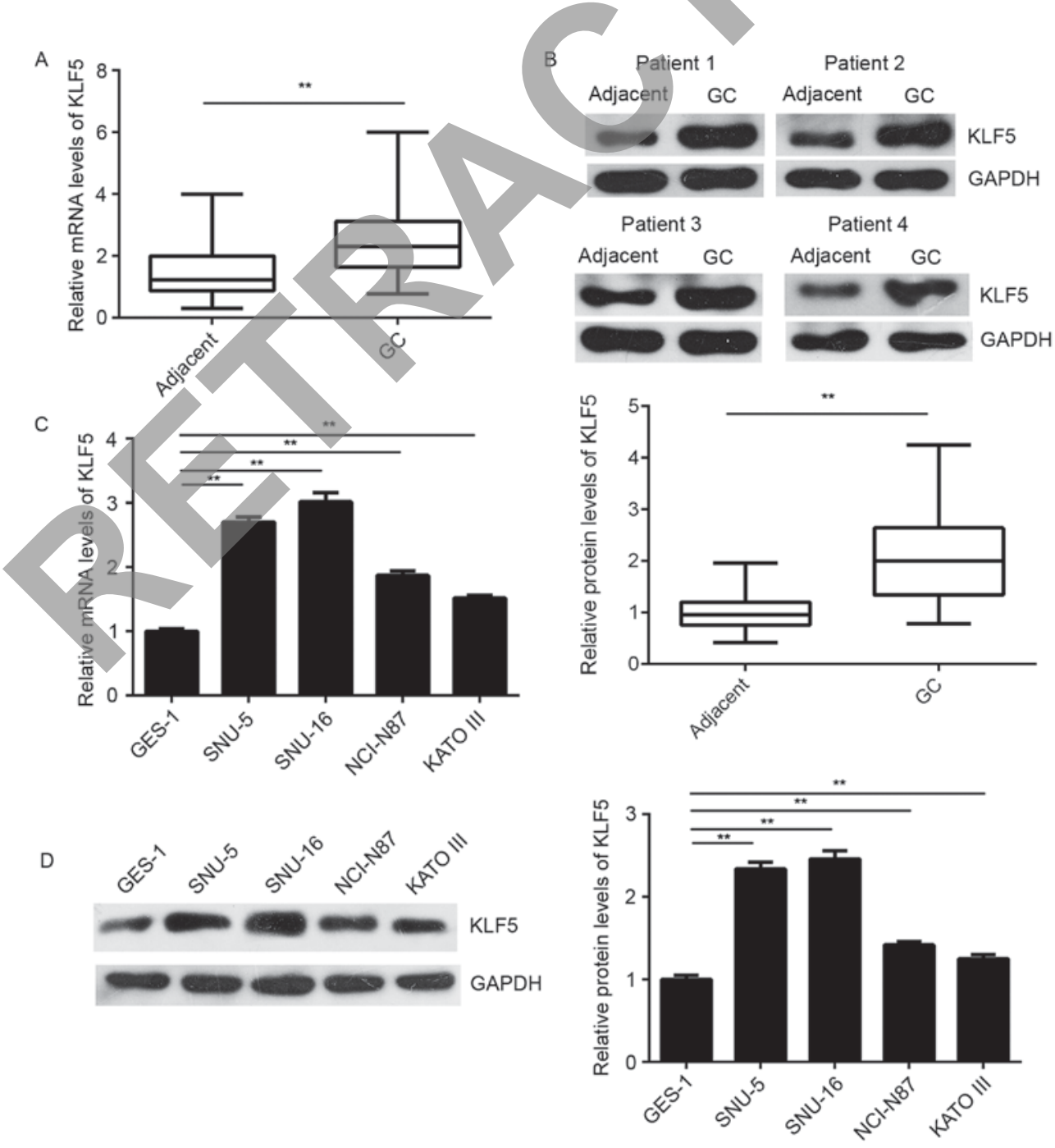

Figure 4. KLF5 is upregulated in GC tissues and cells. (A) RT-qPCR and (B) western blot analysis were conducted to examine the mRNA and protein expression levels of KLF5 in GC tissues and adjacent non-tumor tissues. (C) RT-qPCR and (D) western blot analysis were conducted to examine the mRNA and protein levels of KLF5 in GC cell lines (SNU-5, NCI-N87, SNU-16 and SNU-5) and normal gastric mucosa epithelial GES-1 cells. ${ }^{* *} \mathrm{P}<0.01$. KLP5, Kruppel-like factor 5; GC, gastric cancer; RT-qPCR, reverse transcription-quantitative polymerase chain reaction. 
A

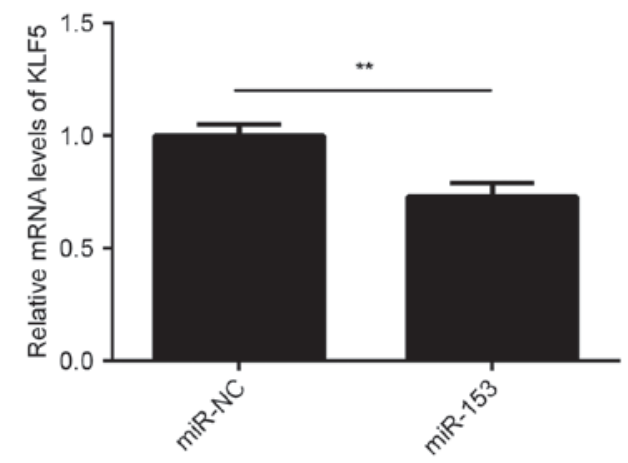

C

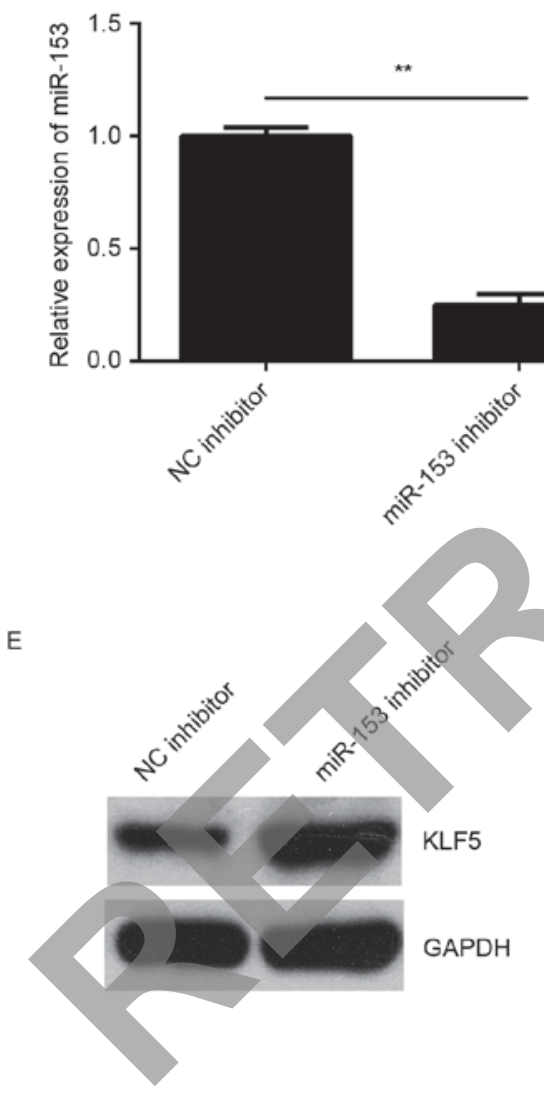

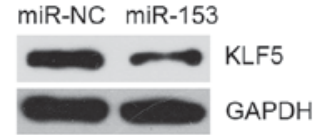

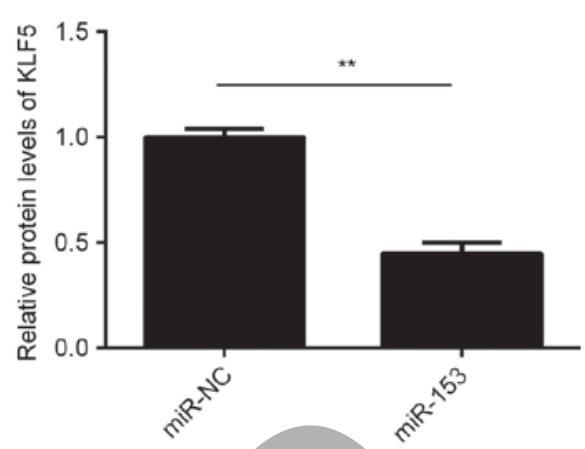

D
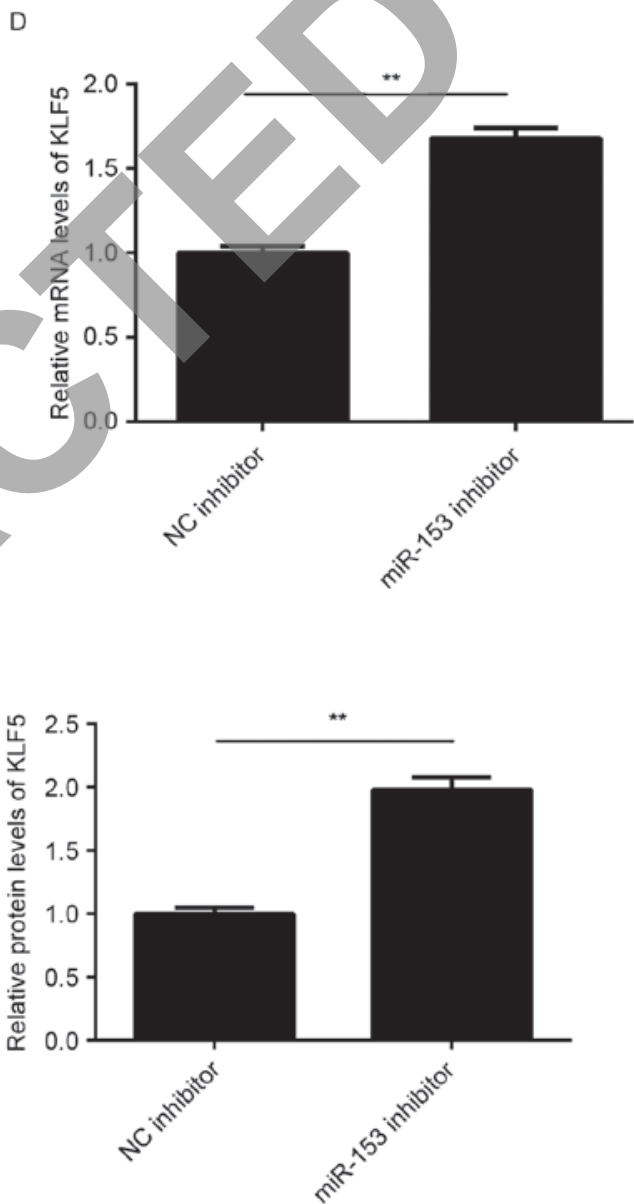

Figure 5. KLF5 is negatively regulated by miR-153 in SNU-5 cells. (A) RT-qPCR and (B) western blot analysis were conducted to examine the mRNA and protein levels of KLF5 in SNU-5 cells transfected with miR-153 mimic or miR-NC. RT-qPCR was conducted to examine the (C) miR-153 levels and (D) KLF5 mRNA levels in SNU-5 cells transfected with miR-153 inhibitor or NC inhibitor. (E) Western blot analysis was also used to determine the protein level of KLF5 in SNU-5 cells transfected with miR-153 or NC inhibitors. ** $\mathrm{P}<0.01$. KLP5, Kruppel-like factor 5; RT-qPCR, reverse transcription-quantitative polymerase chain reaction; miR, microRNA; NC, negative control.

gene of miR-153, and its expression was negatively regulated by miR-153 in SNU-5 cells. Furthermore, overexpression of KLF5 impaired the inhibitory effects of miR-153 on the malignant phenotypes of SNU-5 cells.

In recent years, numerous miRs have been demonstrated to be significantly deregulated and serve promoting or tumor suppressive roles in $\operatorname{GC}(7,9,23)$. For instance, miR-23a promotes the growth in GC cells via directly targeting metallothionein 2A (24), while miR-29a suppresses the growth and invasion of GC cells in vitro by targeting vascular endothelial growth factor A (VEGF-A) (25). In the present study, it was observed that miR-153 was significantly upregulated in GC tissues and cell lines, when compared with matched adjacent non-tumor tissues or normal gastric epithelial cells, respectively. This reduced expression of miR-153 was significantly associated with lymph node metastasis and advanced clinical stage in GC, as well as with shorter survival time of GC patients, consistent with the observations of a previous 


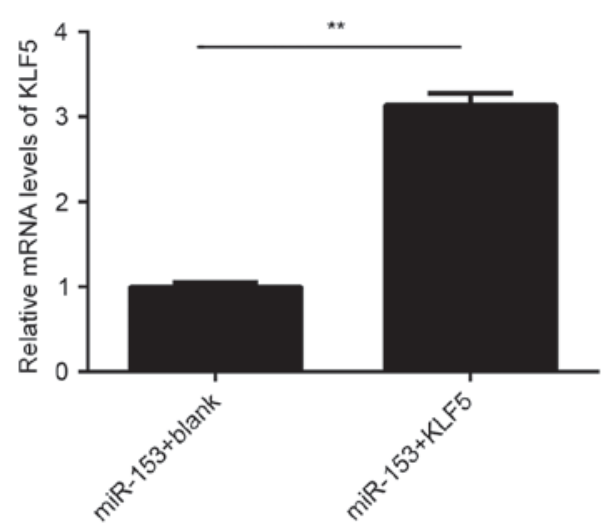

C

D

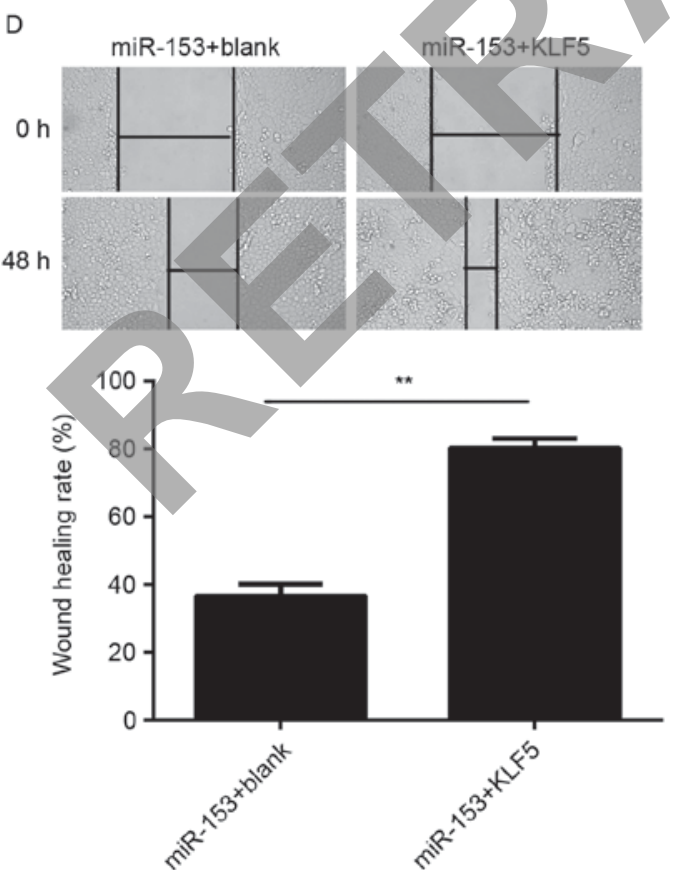

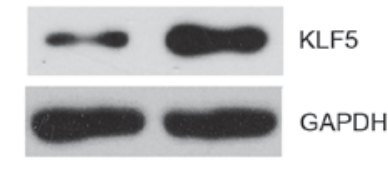
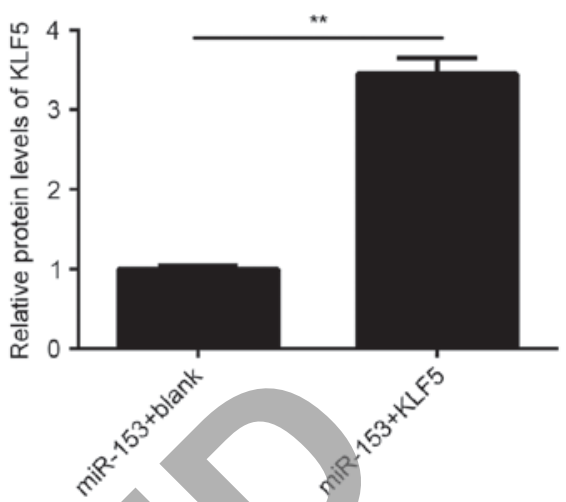

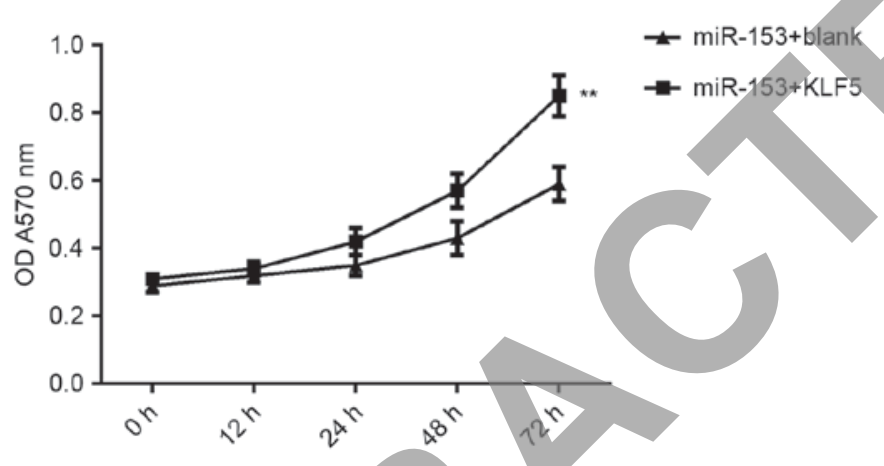

E
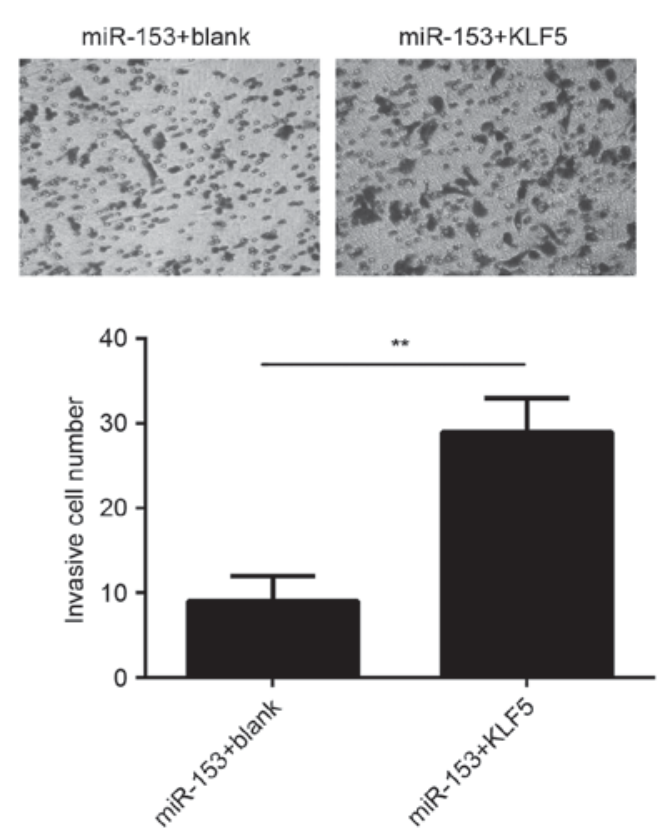

Figure 6. KLF5 is a downstream effector in the miR-153-mediated malignant phenotypes of GC cells. SNU-5 cells were co-transfected with miR-153 mimic and pcDNA3.1-KLF5 plasmid, or co-transfected with miR-153 mimic and blank pcDNA3.1 vector. (A) Reverse transcription-quantitative polymerase chain reaction and (B) western blot analysis were used to examine the mRNA and protein expression levels of KLF5 in the transfected cells. (C) MTT, (D) wound healing and (E) transwell assays were conducted to examine the proliferation, migration and invasion of transfected SNU-5 cells. Magnification: Wound healing assay, $\mathrm{x} 40$; transwell assay, $\mathrm{x} 400 .{ }^{* *} \mathrm{P}<0.01$. KLP5, Kruppel-like factor 5; miR, microRNA; GC, gastric cancer.

study (13). In addition, Zhang et al (13) performed multivariate Cox regression analysis and identified that miR-153 was an independent prognostic marker in GC. Based on these previous findings and the present study observations, it is suggested that 
miR-153 downregulation may contribute to GC progression and the poor outcomes of treatment.

The current study also observed that overexpression of miR-153 markedly reduced the proliferation, migration and invasion of SNU-5 cells. Similarly, Zhang et al (13) reported that miR-153 upregulation was able to inhibit the migration and invasion of GC MKN-45 cells, while knockdown of miR-153 promoted GC SGC-7901 cell migration and invasion. Besides, the authors reported that miR-153 overexpression reduced the expression of SNAI1 and subsequently inhibited the epithelial-mesenchymal transition of GC cells with upregulated E-cadherin and downregulated vimentin (13).

As miRs function through the regulation of their target genes, the potential targets of miR-153 in GC were further investigated. Bioinformatics prediction and dual-luciferase reporter gene assay data confirmed that KLF5 was a direct target gene of miR-153. KLF5 is an important transcription factor, localizing to the nucleus and binding to the epidermal growth factor response element (17). Additionally, KLF5 has been demonstrated to be regulated by post-translational modification by miRs and to function downstream of multiple signaling pathways associated with cell proliferation and motility $(19,26)$. In recent years, the oncogenic role of KLF5 has gradually been revealed in certain common types of human cancer $(20,27)$. For instance, KLF5 promotes cell migration and angiogenesis in bladder cancer by increasing the expression of FYN and VEGF-A $(28,29)$. Besides KLF5 promotes the proliferation, migration and invasion of breast cancer cells by enhancing TNFAIP2 expression (27). Recently, Helicobacter pylori, a pathogenic factor of GC, were found to promote KLF5 expression in gastric epithelial cells in vitro and in vivo (30). Furthermore, nuclear staining of KLF5 expression was reported to be significantly associated with a higher tumor grade, advanced clinical stage, lymph node status and lower disease-free survival rate of GC patients (18). In the current study, the results revealed that KLF5 was significantly upregulated in GC tissues and cell lines, and was negatively regulated by miR-153 in GC cells, which suggests that the reduced expression of miR-153 may contribute to the increased expression of KLF5 in GC. It was further observed that overexpression of KLF5 impaired the suppressive effects of miR-153 on GC cell proliferation, migration and invasion, suggesting that miR-153 has suppressive effects on GC cell proliferation, migration and invasion, at least partly, via targeting KLF5. A similar study also reported that miR-153 inhibited the proliferation and invasion of laryngeal squamous cell carcinoma by targeting KLF5 (26).

In conclusion, the present study demonstrated for the first time that miR-153, which is significantly downregulated in GC, functions as a tumor suppressor, at least partly, via targeting KLF5. Thus, the current study expanded the understanding on the miR-153/KLF5 function in human cancer. Accordingly, these findings suggest that miR-153 may be used as a promising therapeutic candidate for GC treatment.

\section{Competing interests}

The authors declare that they have no competing interests.

\section{References}

1. Siegel RL, Miller KD and Jemal A: Cancer statistics, 2015. CA Cancer J Clin 65: 5-29, 2015.

2. Torre LA, Bray F, Siegel RL, Ferlay J, Lortet-Tieulent J and Jemal A: Global cancer statistics, 2012. CA Cancer J Clin 65: 87-108, 2015.

3. Rocken C: Molecular classification of gastric cancer. Expert Rev Mol Diagn 17: 293-301, 2017

4. Tran P, Nguyen C and Klempner SJ: Targeting the phosphatidylinositol-3-kinase pathway in gastric cancer: Can omics improve outcomes? Int Neurourol J 20 (Suppl 2): S131-S140, 2016.

5. Ambros V: The functions of animal microRNAs. Nature 431: 350-355, 2004

6. Zhu K, He Y, Xia C, Yan J, Hou J, Kong D, Yang Y and Zheng G: MicroRNA-15a inhibits proliferation and induces apoptosis in CNE1 nasopharyngeal carcinoma cells. Oncol Res 24: 145-151, 2016.

7. Wang G, Fu Y, Liu G, Ye Y and Zhang X: miR-218 inhibits proliferation, migration, and EMT of gastric cancer cells by targeting WASF3. Oncol Res 25: 355-364, 2017.

8. Liu X, Li J, Yu Z, Sun R and Kan Q: MiR-935 promotes liver cancer cell proliferation and migration by targeting SOX7. Oncol Res 25: 427-435, 2017.

9. Ji S, Zhang B, Kong Y, Ma F and Hua Y: MiR-326 inhibits gastric cancer cell growth through downregulating NOB1. Oncol Res 25: 853-861, 2017

10. Feng R, Chen X, Yu Y, Su L, Yu B, Li J, Cai Q, Yan M, Liu B and Zhu Z: miR-126 functions as a tumour suppressor in human gastric cancer. Cancer Lett 298: 50-63, 2010.

11. Gao P, Xing AY, Zhou GY, Zhang TG, Zhang JP, Gao C, Li H and Shi DB: The molecular mechanism of microRNA-145 to suppress invasion-metastasis cascade in gastric cancer. Oncogene 32: 491-501, 2013.

. Deng J, Lei W, Xiang X, Zhang L, Yu F, Chen J, Feng M and Xiong J: MicroRNA-506 inhibits gastric cancer proliferation and invasion by directly targeting Yapl. Tumour Biol 36: 6823-6831, 2015.

13. Zhang Z, Sun J, Bai Z, Li H, He S, Chen R and Che X: MicroRNA-153 acts as a prognostic marker in gastric cancer and its role in cell migration and invasion. Onco Targets Ther 8: 357-364, 2015

14. Liu R, Shi P, Nie Z, Liang H, Zhou Z, Chen W, Chen H, Dong C, Yang R, Liu S and Chen C: Mifepristone suppresses basal triple-negative breast cancer stem cells by down-regulating KLF5 expression. Theranostics 6: 533-544, 2016.

15. Wu X, Li L, Li Y and Liu Z: MiR-153 promotes breast cancer cell apoptosis by targeting HECTD3. Am J Cancer Res 6: 1563-1571, 2016.

16. Wang $\mathrm{Z}$ and Liu C: MiR-153 regulates metastases of gastric cancer through Snail. Tumour Biol 2015 [Epub ahead of print].

17. Gao Y, Ding Y, Chen H and Zhou J: Targeting Kruppel-like factor 5 (KLF5) for cancer therapy. Curr Top Med Chem 15: 699-713, 2015.

18. Soon MS, Hsu LS, Chen CJ, Chu PY, Liou JH, Lin SH, Hsu JD and Yeh KT: Expression of Kruppel-like factor 5 in gastric cancer and its clinical correlation in Taiwan. Virchows Arch 459: 161-166, 2011.

19. Jiang Z, Zhang Y, Cao R, Li L, Zhong K, Chen Q and Xiao J: MiR-5195-3p inhibits proliferation and invasion of human bladder cancer cells by directly targeting oncogene KLF5. Oncol Res 25: 1081-2587, 2017.

20. Chia NY, Deng N, Das K, Huang D, Hu L, Zhu Y, Lim KH, Lee MH, Wu J, Sam XX, et al: Regulatory crosstalk between lineage-survival oncogenes KLF5, GATA4 and GATA6 cooperatively promotes gastric cancer development. Gut 64: 707-719, 2015.

21. Yegin EG and Duman DG: Staging of esophageal and gastric cancer in 2014. Minerva Med 105: 391-411, 2014.

22. Livak KJ and Schmittgen TD: Analysis of relative gene expression data using real-time quantitative PCR and the 2(-Delta Delta C(T)) method. Methods 25: 402-408, 2001.

23. Li C, Lu S and Shi Y: MicroRNA-187 promotes growth and metastasis of gastric cancer by inhibiting FOXA2. Oncol Rep 37: 1747-1755, 2017.

24. An J, Pan Y, Yan Z, Li W, Cui J, Yuan J, Tian L, Xing R and Lu Y: MiR-23a in amplified 19p13.13 loci targets metallothionein 2A and promotes growth in gastric cancer cells. J Cell Biochem 114: 2160-2169, 2013 
25. Chen L, Xiao H, Wang ZH, Huang Y, Liu ZP, Ren $H$ and Song H: miR-29a suppresses growth and invasion of gastric cancer cells in vitro by targeting VEGF-A. BMB Rep 47: 39-44, 2014.

26. Liu JY, Lu JB and Xu Y: MicroRNA-153 inhibits the proliferation and invasion of human laryngeal squamous cell carcinoma by targeting KLF5. Exp Ther Med 11: 2503-2508, 2016.

27. Jia L, Zhou Z, Liang H, Wu J, Shi P, Li F, Wang Z, Wang C, Chen $\mathrm{W}$, Zhang $\mathrm{H}$, et al: KLF5 promotes breast cancer proliferation, migration and invasion in part by upregulating the transcription of TNFAIP2. Oncogene 35: 2040-2051, 2016.

28. Du C, Gao Y, Xu S, Jia J, Huang Z, Fan J, Wang X, He D and Guo P: KLF5 promotes cell migration by up-regulating FYN in bladder cancer cells. FEBS Lett 590: 408-418, 2016.
29. Gao Y, Wu K, Chen Y, Zhou J, Du C, Shi Q, Xu S, Jia J, Tang X, Li F, et al: Beyond proliferation: KLF5 promotes angiogenesis of bladder cancer through directly regulating VEGFA transcription. Oncotarget 6: 43791-43805, 2015.

30. Noto JM, Khizanishvili T, Chaturvedi R, Piazuelo MB, Romero-Gallo J, Delgado AG, Khurana SS, Sierra JC, Krishna US, Suarez G, et al: Helicobacter pylori promotes the expression of Krüppel-like factor 5, a mediator of carcinogenesis, in vitro and in vivo. PLoS One 8: e54344, 2013.

(i) (9) This work is licensed under a Creative Commons Attribution-NonCommercial-NoDerivatives 4.0 International (CC BY-NC-ND 4.0) License. 